\title{
What Is the Role for Patelloplasty With Gullwing Osteotomy in Revision TKA?
}

\author{
Jeremy M. Gililland MD, Presley Swann MD, Christopher E. Pelt MD, \\ Jill Erickson PA, Nadia Hamad MSc, ATC, Christopher L. Peters MD
}

Published online: 30 May 2015

(C) The Association of Bone and Joint Surgeons (B) 2015

\begin{abstract}
Background Management of the patella in revision total knee arthroplasty (TKA) is challenging as a result of the deficient or unusable bone stock for patellar resurfacing that is frequently encountered. Options proposed in this setting include various patelloplasty procedures, patellectomy, and special patellar components. We sought to better define the role and results of one patelloplasty procedure, the gullwing osteotomy, used in revision TKA.
\end{abstract}

One of the authors certifies that he (CLP), or a member of his immediate family, has or may receive payments or benefits, during the study period, an amount of USD 100,001 to USD 1,000,000 from Biomet, Inc (Warsaw, IN, USA). One the authors certifies that he (CEP), or a member of his immediate family, has or may receive payments or benefits, during the study period, an amount of USD 10,000 to USD 100,000 from Biomet, Inc. One the authors certifies that he (JMG), or a member of his immediate family, has or may receive payments or benefits associated with institutional research support during this study period an amount of USD 100,001 to USD $1,000,000$ from Biomet, Inc.

All ICMJE Conflict of Interest Forms for authors and Clinical Orthopaedics and Related Research ${ }^{\circledR}$ editors and board members are on file with the publication and can be viewed on request.

Clinical Orthopaedics and Related Research ${ }^{\mathbb{R}}$ neither advocates nor endorses the use of any treatment, drug, or device. Readers are encouraged to always seek additional information, including FDAapproval status, of any drug or device prior to clinical use. Each author certifies that his or her institution approved the human protocol for this investigation and that all investigations were conducted in conformity with ethical principles of research.

J. M. Gililland, P. Swann, C. E. Pelt, J. Erickson, N. Hamad,

C. L. Peters $(\bowtie)$

Department of Orthopaedics, University of Utah, 590 Wakara

Way, Salt Lake City, UT 84108, USA

e-mail: chris.peters@hsc.utah.edu
Questions/purposes (1) How much improvement in the outcome measures of range of motion and Knee Society scores was seen after revision TKA with a gullwing osteotomy? (2) What are the radiographic results of this osteotomy as judged by patellar healing and patellar tracking? (3) What complications are associated with the gullwing osteotomy in revision TKA?

Methods Between December 2003 and July 2012, we used a gullwing osteotomy on patients undergoing revision TKA $(n=238)$ in which the patellar remnant was avascular or less than $12 \mathrm{~mm}$ thick. This uncommon procedure was used in 17 of $115(15 \%)$ of the patellae revised during this time. We performed manual chart reviews on all patients to collect preoperative and postoperative range of motion and Knee Society scores as well as radiographic review at last followup to assess patellar healing and tracking.

Results In patients with at least 2 years of followup, the preoperative range of motion was a median $-7.5^{\circ}$ of extension (interquartile range $[\mathrm{IQR}],-15^{\circ}-0^{\circ}$ ) and $90^{\circ}$ of flexion (IQR, $\left.90^{\circ}-100^{\circ}\right)$. Postoperative extension improved to $0^{\circ}$ (IQR, $0^{\circ}-0^{\circ} ; \mathrm{p}=0.015$ ). With the numbers available, median flexion arc did not change at last followup $\left(110^{\circ}\right.$; IQR, $95^{\circ}-$ $\left.120^{\circ} ; \mathrm{p}=0.674\right)$. The Knee Society score improved from a combined (clinical + functional) mean of 86 (95\% confidence interval [CI], 56-116) preoperatively to 142 (95\% CI, 121$163 ; \mathrm{p}<0.001$ ) postoperatively. Radiographically, 12 of 13 patients demonstrated healing of the osteotomy with osseous union and one patient healed with a fibrous union. Nine of the 10 patients with at least 2 years of followup had a centrally tracking gullwing osteotomized patella at last followup. One patient, with just over 3 years of followup, exhibited lateral subluxation without evidence of fracture. Three of the 10 patients with greater than 2 years of followup developed recurrent infections. One patient had avascular necrosis with fragmentation of the patella at 4 months postoperatively. 
Conclusions Patellar bone stock is often compromised in revision TKA, leaving the surgeon with very few options for reconstruction. Using this technique, we found acceptable function, no aseptic rerevisions for patellofemoral complications, nine of 10 of patellae tracking within the trochlear groove, and radiographic healing of the majority of the osteotomies. The gullwing osteotomy may be considered an option in these difficult revisions, but further studies with more complete followup are needed.

Level of Evidence Level IV, therapeutic study.

\section{Introduction}

The number of patients undergoing revision TKA is expected to double in the next 8 years [7]. Although maintaining the patellar component is ideal for preservation of bone stock, its removal is sometimes necessary. The reconstruction of a patella that is too thin or avascular to resurface with a standard cemented or cementless component remains challenging and many treatment options exist. The goals of reconstruction include stable fixation (if repeat resurfacing is performed) as well as both maintaining and maximizing the extensor mechanism. Maintaining the extensor mechanism is achieved by not disrupting the integrity of the connection between the quadriceps and patellar tendons. Maximizing the extensor mechanism occurs when the lever arm created by the patella is kept intact thus avoiding a patellectomy, which inherently decreases the effect of the lever arm of the extensor mechanism. Current treatment options include patella bone grafting, patelloplasty, patellectomy, onlay cementation, biconvex patellar prosthesis, tantalum patellar prosthesis, and the gullwing osteotomy [1, 4, 6, 8-10].

The gullwing osteotomy was first described in 1999 at an annual meeting of hip and knee surgeons [10]. Vince et al. [10] described the gullwing osteotomy as a successful method for treating the deficient patella when further implant revision is not feasible. The gullwing osteotomy is a sagittal osteotomy made in the articulating surface of the patella, converting a concave thin patellar shell into a $\mathrm{V}$-shaped patella. The main proposed benefit is improved tracking of the patellar remnant and less point loading of the outer edge of the patellar bone, which may decrease anterior knee pain. The concern with any osteotomy of the patella would be avascular necrosis or nonunion as well as extensor mechanism disruption and resultant extensor lag or weakness. However, no case of nonunion or extensor mechanism disruption has been reported in the limited published literature regarding the gullwing osteotomy [6, 10]. In his original series of four patients, Vince et al. [10] demonstrated $100 \%$ healing and centrally tracking patellae after the procedure with improved outcomes both clinically and radiographically. Reviewing a series of 12 patients, Klein et al. [6] also reported that the gullwing osteotomy was an effective method for salvaging the deficient patella with $100 \%$ bony union, $100 \%$ central tracking, and statistically improved pain scores. With the limited research published on this topic and the increasing number of knee revisions expected in the future, we sought to review our surgical database for a single surgeon's experience with gullwing osteotomy.

Specifically we sought to answer the following questions: (1) How much improvement in the outcome measures of ROM and Knee Society scores was seen after revision TKA with a gullwing osteotomy? (2) What are the radiographic results of this osteotomy as judged by patellar healing and patellar tracking? (3) What complications are associated with the gullwing osteotomy in revision TKA?

\section{Patients and Methods}

After receiving an exemption from the institutional review board for secondary data analysis, we performed a retrospective study on a series of 17 patients who underwent revision TKA with a gullwing osteotomy (Fig. 1) between December 2003 and July 2012. Patients were identified using a surgical database of the senior author (CLP). No gullwing osteotomy cases were excluded. During this time period, we identified 204 patients who underwent 238 revision TKAs (excluding simple polyethylene exchanges). Of the 238 revision TKAs, 115 patellae were revised: 80 underwent resurfacing, 17 were those who received the gullwing osteotomy, 11 were treated with patellectomy, and seven had patellectomy with tubularization. The

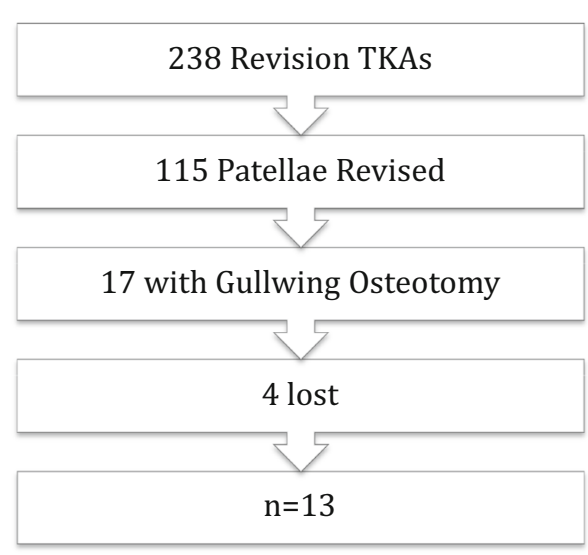

Fig. 1 This flow diagram demonstrates the number of revision TKAs performed during this time period, the number of gullwing osteotomies, and ultimately the number of patients included in the series. 
indication for the gullwing osteotomy was when a thin or avascular patella of typically less than $12 \mathrm{~mm}$ thickness was encountered. In general these patellae are a thin concave cortical shell that have the following characteristics: (1) the patella tracks eccentrically in the trochlear groove; and (2) there is point contact on the concave cortical shell rim that may be a potential source of pain.

The gullwing osteotomy converts a thin concave cortical shell into a V-shaped patella remnant, which has a better chance of tracking centrally in the trochlear groove. Other methods available during this time period included a porous metal patella, bone grafting, and patellectomy. We have no experience with the porous metal patella and patellectomy was reserved for completely avascular small patellar remnants.

Of the 17 patients, four (24\%) were ultimately deemed lost to followup because they failed to return for a minimum 2-year followup (Fig. 1). Two of these four were last seen at approximately 6 months; however, they failed to return after that. Both of these patients died at roughly 4 and 8 years after the procedure. The other two were last seen 6 weeks after the procedure and have failed to return. The 13 remaining patients demonstrated a mean followup of 4.3 years (range, 6 weeks to 8 years). Three of the 13 patients died before their 1-year followup. However, the osteotomy on these patients appeared healed and in good position at last followup and they were retained in the series. Two additional patients died at 4 and 7 years after the procedure and had approximately 3 and 6 years of followup, respectively. The remaining eight patients have all been seen within the last 2 years with a mean followup of 5.8 years (range, 2-8 years).

Manual chart reviews were performed on all patients to collect data regarding clinical and radiographic outcomes as well as complications. The preoperative diagnosis was infection in 14 patients, two patients had aseptic failure of cemented implants, and one patient had aseptic failure of cementless implants. Patella deficiency was seen in all patients and the preoperative mean patellar thickness was $11 \mathrm{~mm}$ (range, 9-17 $\mathrm{mm}$ ). One patient with a preoperative patellar thickness of $17 \mathrm{~mm}$ was unable to receive another patella component as a result of central bone loss from prior surgery. Another patella with $15 \mathrm{~mm}$ of preoperative thickness was found to have significant wear and once the cement was removed was too thin for reimplantation. The mean age of our series was 65 years (range, 49-85 years) with seven of 13 being men. The mean body mass index was $35 \mathrm{~kg} / \mathrm{m}^{2}$ (range, 22-51 kg/m ${ }^{2}$ ).

All procedures were performed at a tertiary referral center and the gullwing osteotomy was performed similarly to the method described by Klein et al. [6]. A medial parapatellar arthrotomy was used in 15 (88\%) of the cases and a quadriceps snip approach was used in the remaining
$12 \%$ (two of 17). The patellar surface was débrided of all nonviable tissue and a midline sagittal osteotomy was performed from the deep surface of the patella directed toward the superficial surface. Special care was taken to ensure that the anterior soft tissue sleeve remained intact. The osteotomy was performed with an oscillating saw initially and completed with an osteotome. Tibial and femoral revision components were used in all cases and the level of constraint varied with a posterior stabilized bearing in one of 13, a varus-valgus constrained bearing in 11 of 13 , and a hinged articulation in one of 13. Postoperative recovery included a knee immobilizer for the first 2 postoperative days while the continuous femoral nerve block remained in place with both active and passive ROM as tolerated allowed after that time period. Patients were made $25 \%$ partial weightbearing for the first 6 weeks postoperatively with an ambulatory assistive device (walker or crutches) and then allowed to advance to weightbearing as tolerated after that. This postoperative regimen is consistent with our standard regimen for revision TKAs and we did not alter this for these patients with gullwing osteotomies.

We assessed improvement in clinical outcomes by evaluating the pre- and postoperative differences in knee ROM (flexion and extension) and the combined (clinical + functional) Knee Society scores (KSS) from the previous version of the $\operatorname{KSS}[2,3,5]$. Additionally, we reviewed the postoperative presence of extensor lag and the need for ambulatory aids as indicators for less favorable outcomes.

Radiographically, as per our clinical standard of care, patients received pre- and postoperative AP, lateral, and sunrise views. Radiographs were reviewed by an experienced clinician (JE) and were used to assess for union of the osteotomy, evidence of lateral subluxation or fracture, and patellar tracking. A patella was considered to be tracking centrally when the majority of the patella was located centrally within the trochlear groove (Fig. 2). Lateral tracking (lateral subluxation) of the patella was defined as when the majority of the patella tracked laterally over the lateral condyle on the sunrise view (Fig. 3). The patella was considered healed with an osseous union when there was solid ossification across the osteotomy with no radiolucent lines. A fibrous union was defined as radiolucency through the osteotomy that failed to heal with ossification.

Complications were recorded from the electronic medical record. We reviewed the records for evidence of operative knee complications including reinfection and reoperation for any reason. The Social Security Death Index was used to assess mortality.

Descriptive statistics are reported as means (95\% confidence intervals [CIs]) for continuous data and median (interquartile range [IQR]) for categorical or nonparametric 


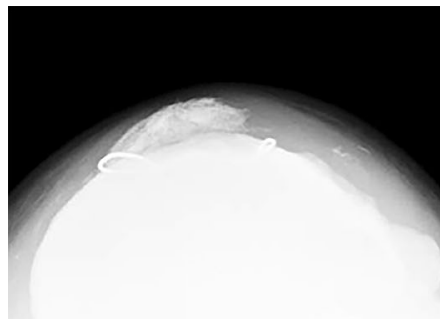

Before
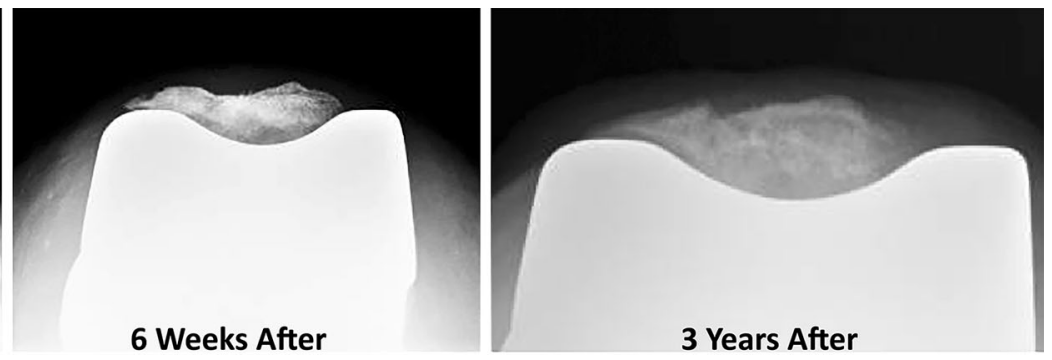

Fig. 2 In this sunrise view, the patella is seen preoperatively, 6 weeks after the index gullwing osteotomy, and 3 years after the index procedure.

Fig. 3 These images demonstrate lateral tracking (lateral subluxation) of the patella both before and 6 weeks after with a healed patella after gullwing osteotomy.
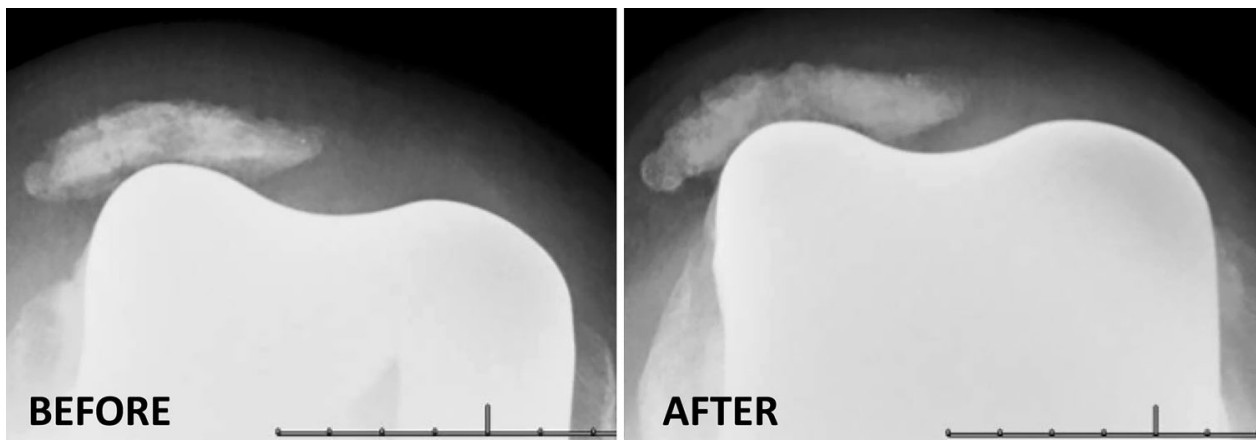

data. Pre- and postoperative measurements were compared using the paired-sample t-tests or the Wilcoxon signedrank test when the data showed a nonparametric distribution (Shapiro-Wilk test).

\section{Results}

Knee ROM and the combined KSS improved in the patients with at least 2 years of followup $(n=10)$. The preoperative ROM was a median $7.5^{\circ}$ lack of full extension (IQR, $15^{\circ}-0^{\circ}$ ) and $90^{\circ}$ of flexion (IQR, $90^{\circ}-100^{\circ}$ ). Postoperative extension improved to $0^{\circ}\left(\mathrm{IQR}, 0^{\circ}-0^{\circ} ; \mathrm{p}=\right.$ $0.015)$. With the numbers available, the median active flexion arc did not change at last followup (median $110^{\circ}$; $\left.\mathrm{IQR}, 95^{\circ}-120^{\circ} ; \mathrm{p}=0.674\right)$. The KSS improved from a combined mean of 86 (95\% CI, 56-116) preoperatively to $142(95 \%$ CI, 121-163; p < 0.001) postoperatively. One patient reported a worse KSS score at nearly 6 years of followup from a preoperative score of 154 to a postoperative score of 130 . Only two of the patients demonstrated postoperative extensor lags. One patient did not have a preoperative lag and on postoperative followup showed $5^{\circ}$ of extensor lag. The other patient had a preoperative lag of $70^{\circ}$ and improved to a postoperative lag of $15^{\circ}$. The patient with a preoperative extensor lag of $70^{\circ}$ had multiple prior procedures likely contributing to the complexity of this case. However, the extensor mechanism remained intact, as noted by the postoperative improvement, and it seems likely that this extensor lag was pain-mediated preoperatively. At last followup, six of 10 patients required assistive devices for ambulation; four required a cane, one used a walker, and one was limited to a wheelchair.

Radiographically, 12 of 13 patients demonstrated healing of the osteotomy with osseous union and one patient healed with a fibrous union (Fig. 4). Nine of the 10 patients with at least 2 years of followup had a centrally tracking gullwing osteotomized patella at last followup (Fig. 2). The other patient, with just over 3 years of followup, exhibited lateral subluxation without evidence of fracture (Fig. 3). The mean postoperative patellar thickness was 9 mm (95\% CI, 8-11) compared with a preoperative patellar thickness of $11 \mathrm{~mm}$ (95\% CI, 10-13; p = 0.042).

Three of the 10 patients with greater than 2 years of followup developed recurrent infections, two of which were treated successfully with repeat two-stage revision TKA. The other patient ended treatment with an arthrodesis. There were no other revisions or reoperations and, perhaps most importantly, no extensor mechanism failures. One patient, with approximately 7 years of followup, had avascular necrosis with fragmentation of the patella noted at 4 months postoperatively; however, the extensor mechanism continued to function well (Fig. 5).

\section{Discussion}

Management of the patella in revision TKA is challenging as a result of frequently encountered deficient and/or unusable bone stock for patellar resurfacing. The goals of 

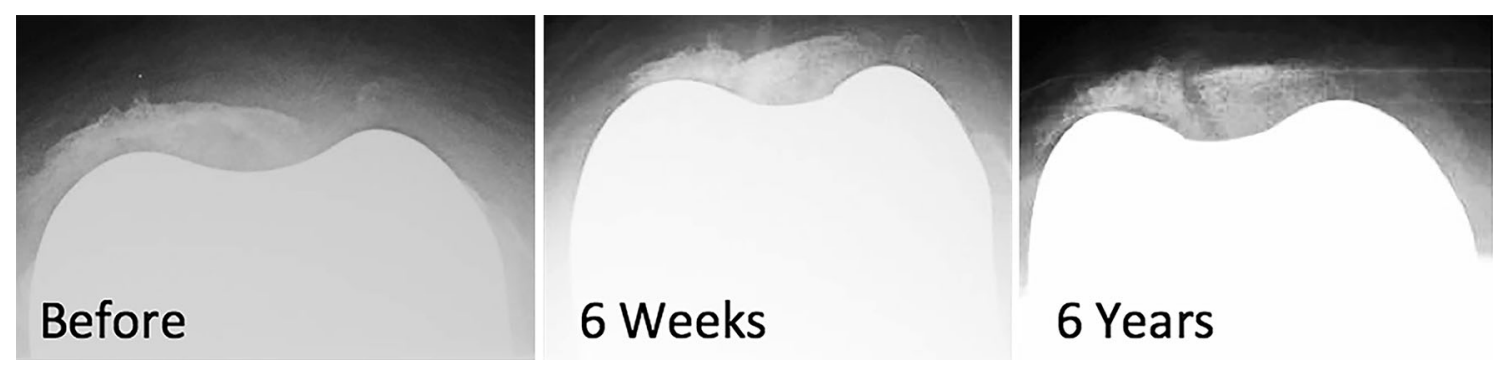

Fig. 4 A fibrous union of the patella is seen in this image 6 years after the index gullwing osteotomy was performed.

Fig. 5A-D This series of images portrays what appears to be a healthier patella preoperatively (A) and immediately after the index procedure (B). However, avascular necrosis of the patella is seen at both the 6-week followup (C) and further at the (D) 4-month followup.
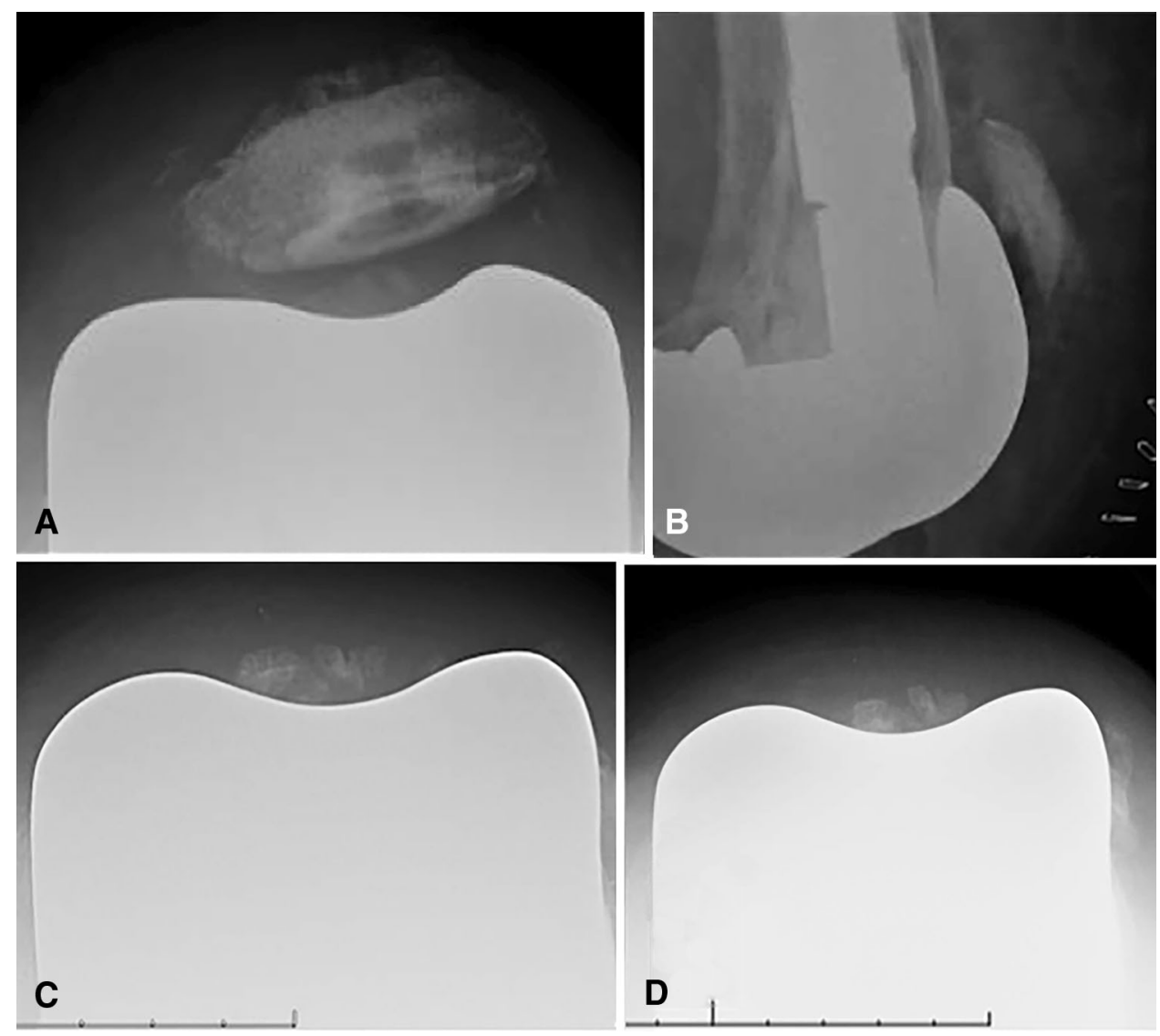

patella reconstruction include stable fixation, maintenance of the extensor mechanism integrity, and maximization of the extensor mechanism function. With the small numbers involved and multiple options available to treat the deficient patella, providing definitive data with prospective randomized studies or even comparative studies is difficult. The purpose of this study was to better define the role and results of one option, the gullwing osteotomy. Our results demonstrate maintenance of ROM including terminal active extension in most patients, good knee scores, healing of the osteotomy in most patients, few complications, and the ability to avoid a patellectomy.
Limitations of this study include the retrospective nature in a limited number of patients that could result in a selection bias. However, we used this procedure consistently during the period in question, yet it is possible that some patients whose bone stock was close to our indications for this procedure ( $<12 \mathrm{~mm}$ thickness) may have been left unresurfaced without a gullwing osteotomy if the surgeon felt this had a chance to heal. It should be noted that we did not use alternatives, like porous metal patellar revision components, and so we believe that this series of 17 patients represents most of our experience with this difficult clinical problem. Another limitation of this study is that of 
transfer bias. A relatively high proportion of the patients were lost to followup before the 2-year minimum (four of 17), and another three died in the first year. It is possible that some of those who were lost to followup have gone on to develop problems or were revised at other centers; the three who died before the minimum followup were noted to have healed their osteotomies. Future studies with more complete followup will need to evaluate this procedure to confirm our findings; multicenter studies will be needed to compare possible approaches to the deficient patella. One final limitation is that this is a noncomparative case series, which does not allow us to compare the gullwing osteotomy to other patellar revision techniques available in our given patient population.

Our series demonstrated improved knee extension after revision TKA with the gullwing osteotomy as well as improvement in clinical and functional outcomes as measured by the KSS. Klein et al. [6] also reported similar findings with an increase in the mean arc of motion from a preoperative arc of $59^{\circ}$ to a postoperative arc of $109^{\circ}$ and improvements in the KSS. Furthermore, we had no evidence of extensor failure and only two patients with a postoperative extensor lag, although one was minimal $\left(5^{\circ}\right)$. The patients' outcome scores improved and only one of 10 exhibited lateral subluxation. However, six of 10 patients with at least 2 years of followup still required an ambulatory assistance device. Barrack et al. [1] reported in 21 patients with revision TKA that the patients left with a bony patellar shell alone versus patellar resurfacing had a poorer outcome demonstrated by lower reported postoperative satisfaction and function. In contrast, Patil et al. [8] compared patellar revision options in 46 total knee revisions in patellar retention (22 patients), resurfacing (13 patients), and patelloplasty (11 patients) groups. This study reported similar KSS and SF-36 scores for all three groups. Furthermore, Rorabeck et al. [9] noted that although not all revisions were patellar bone-deficient, if the surgeon could achieve a centrally tracking patella, better outcomes were obtained compared with a patelloplasty left lateralized.

In our series looking at the radiographic outcomes, 12 of 13 patients demonstrated healing of the osteotomy with osseous union and one patient healed with a fibrous union. Nine of 10 patients, with at least two years of follow up, had a centrally tracking gullwing osteotomy patella at latest followup. One of the 10 patients exhibited lateral subluxation without evidence of fracture. Klein et al. [6] reported that 10 out of 10 patients healed with an osseous union and were tracking centrally with radiographic followup; four had fragmentation, and three showed slight lateral overhang.

In conclusion, all of the gullwing osteotomies in this small series healed either with ossified or fibrous unions and there were no complications directly related to the osteotomy itself. The gullwing osteotomy may be considered as an option in these difficult revisions, but further studies with more complete followup are needed. The gullwing osteotomy appears to offer a relatively straightforward surgical option that maintains the extensor mechanism with minimal morbidity.

Acknowledgments We thank Mike B. Anderson MSc, for his contributions to this manuscript.

\section{References}

1. Barrack RL, Matzkin E, Ingraham R, Engh G, Rorabeck C. Revision knee arthroplasty with patella replacement versus bony shell. Clin Orthop Relat Res. 1998;356:139-143.

2. Dossett HG, Estrada NA, Swartz GJ, LeFevre GW, Kwasman BG. A randomised controlled trial of kinematically and mechanically aligned total knee replacements: two-year clinical results. Bone Joint J. 2014;96:907-913.

3. Dowsey MM, Choong PF. The utility of outcome measures in total knee replacement surgery. Int $J$ Rheumatol. 2013;2013:506518.

4. Garcia RM, Kraay MJ, Conroy-Smith PA, Goldberg VM. Management of the deficient patella in revision total knee arthroplasty. Clin Orthop Relat Res. 2008;466:2790-2797.

5. Insall JN, Dorr LD, Scott RD, Scott WN. Rationale of the Knee Society clinical rating system. Clin Orthop Relat Res. 1989;248:13-14.

6. Klein GR, Levine HB, Ambrose JF, Lamothe HC, Hartzband MA. Gull-wing osteotomy for the treatment of the deficient patella in revision total knee arthroplasty. $J$ Arthroplasty. 2010;25:249-253.

7. Kurtz S, Ong K, Lau E, Mowat F, Halpern M. Projections of primary and revision hip and knee arthroplasty in the United States from 2005 to 2030. J Bone Joint Surg Am. 2007;89:780785.

8. Patil N, Lee K, Huddleston JI, Harris AH, Goodman SB. Patellar management in revision total knee arthroplasty: is patellar resurfacing a better option? J Arthroplasty. 2010;25:589-593.

9. Rorabeck CH, Mehin R, Barrack RL. Patellar options in revision total knee arthroplasty. Clin Orthop Relat Res. 2003;416:84-92.

10. Vince K, Blackburn D, Ortaaslan S, Thadani P. 'Gull-wing' osteotomy of the patella in total knee arthroplasty. American Association of Hip and Knee Surgeons Nonth Annual Meeting. Dallas, TX. J Arthroplasty. 1999:254. 